\title{
Optimization of Traffic Flow On KS Tubun Street using Greenberg Model
}

\author{
Embay Rohaeti, Ary Mulyatiningsih
}

\begin{abstract}
KS Tubun Street is a street in Bogor, which has a fairly high vehicle volume and become one of a high-traffic jam area. This is caused by KS Tubun Street is the main road for road users from Jakarta and Bogor. Traffic jam problem that occurs due to the confluence interchange of traffic flow and traffic lights settings that are not proportional to the volume of vehicles across the road. Optimization of traffic flow at KS Tubun Street performed by the stages of forming a model of traffic flow, determining the density and velocity of the vehicle is based on the Greenberg model, and determining the length of the traffic lights to avoid a buildup of vehicles. The result is a traffic flow model with distance and time parameters. The density of vehicles that occurs on the streets of KS. Tubun street based on the Greenberg model between 180 to 240 unit car of passanger (ucp) with the average velocity of vehicles 15 to $19.5 \mathrm{~km}$ per hour. The density of vehicles on KS. Tubun street can be break down by increasing time. Traffic light cycle time can be reduced for 8 seconds with the red light glowing time is 80 seconds and the green light glowing time is 62 seconds.
\end{abstract}

Index Terms: Traffic flow, Traffic Lights, Greenberg Model.

\section{INTRODUCTION}

KS. Tubun Street is a street in Bogor with a high volume of vehicles. This area became one of the traffic jam area because it is the main road for the road users from Jakarta and Bogor. Although KS Tubun Street has traffic lights, but the road is still one of the roads that have high levels in traffic jam. The traffic jam occurs because of the abundance of vehicles entered in the line of traffic light imbalancedwith the number of vehicles going out the line, so it's needed to analyze in determining of waiting time of vehicle in the line of the traffic light that can reduce the buildup of vehicles on KS. Tubun Street. The relationship of velocity and density can be expressed in the form of the exponential through the Greenberg model that explained the flow of vehicles moving like water flow and not always stated that if low velocity then increase vehicle density [1]. The goal of this research is to simulate the flow of traffic in the KS Tubun Street and determine the waiting time of vehicles in the line of traffic lights.

Revised Manuscript Received on April 25, 2019.

Embay Rohaeti, Department of Mathematic, Pakuan University, Indonesia.

Aru Mulyatiningsih, Department of Mathematic, Pakuan University, Indonesia.

\section{MATERIALS AND METHODS}

This research uses survey data on Service officer of Traffic and Transportation in Bogor City in 2016 on the flow of traffic in KS. Tubun Street at 06.00 to 12.15 o'clock. The data is converted in accordance to vehicle type MC (motorcycle), which is 0.15 times the number of LV (light vehicles)and it is 1 times the number of $\mathrm{HV}$ (heavy vehicles), that is 1.5 times the number of the vehicles. The results of the conversion can be seen in Table 1 .

Table1. Data of Traffic Flow In KS. Tubun Street.

\begin{tabular}{|c|c|c|c|c|c|c|c|}
\hline Time & MC & LV & HV & Time & MC & LV & HV \\
\hline $06.00-06.15$ & 411 & 87 & 4 & $09.00-09.15$ & 546 & 94 & 4 \\
\hline $06.15-06.30$ & 536 & 91 & 1 & $09.15-09.30$ & 651 & 116 & 5 \\
\hline $06.30-06.45$ & 454 & 121 & 3 & $09.30-09.45$ & 619 & 131 & 10 \\
\hline $06.45-07.00$ & 681 & 149 & 5 & $09.45-10.00$ & 607 & 88 & 8 \\
\hline $07.00-07.15$ & 704 & 167 & 9 & $10.00-10.15$ & 618 & 94 & 12 \\
\hline $07.15-07.30$ & 772 & 188 & 5 & $10.15-10.30$ & 598 & 81 & 11 \\
\hline $07.30-07.45$ & 669 & 167 & 6 & $10.30-10.45$ & 493 & 76 & 6 \\
\hline $07.45-08.00$ & 681 & 119 & 7 & $10.45-11.00$ & 559 & 101 & 8 \\
\hline $08.00-08.15$ & 633 & 134 & 11 & $11.00-11.15$ & 594 & 133 & 13 \\
\hline $08.15-08.30$ & 619 & 122 & 9 & $11.15-11.30$ & 634 & 141 & 2 \\
\hline $08.30-08.45$ & 521 & 146 & 14 & $11.30-11.45$ & 591 & 123 & 4 \\
\hline $08.45-09.00$ & 512 & 102 & 7 & $11.45-12.00$ & 608 & 119 & 6 \\
\hline
\end{tabular}

The Method of optimizing the flow of traffic in KS. Tubun Street by Greenberg model is preceded by converting data in ucp (unit of car passenger), then simulate the model of traffic flow with Greenberg model by MATLAB and determine the waiting time of vehicles in the queue of traffic lights.

\section{RESULT}

\section{A. Converting data in ucpunit (car of passanger)}

Data in table 1 is converted into ucp, and the result can be seen in Table 2.

Based on the table above, the total average of vehicle across KS Tubun Street is 226 ucp per 15 minutes with rush hour at 7.15-7.30 with 311 ucp vehicles that passed by.

\section{B. Simulation of Traffic Flow Model by Greenberg Model}

According to Kachroo et al (2012) Greenberg model assumed that traffic flow has a similarity with fluid flow and the represented as follow: 


\section{Optimization of Traffic Flow On KS Tubun Street Using Greenberg Model}

$v(\rho)=v_{f}\left(\ln \left(\frac{\rho_{\max }}{\rho}\right)\right)$

with $v(\rho)$ is velocity of independent flow ( $\mathrm{km} / \mathrm{hour}$ ), $\rho_{\max }$ is maximum density, $V_{f}$ is velocity of independent flow (km/hour), $\rho$ is vehicle density (ucp/km). The simulation uses $45 \mathrm{~km} /$ hour on velocity of independent flow, $922 \mathrm{~km} /$ hour on maximum density, $10 \mathrm{~km}$ on distance in 60 minutes.

1. Before doing the simulation, based on Greenberg model, first is capture the actual condition of traffic flow in KS Tubun street as follow :

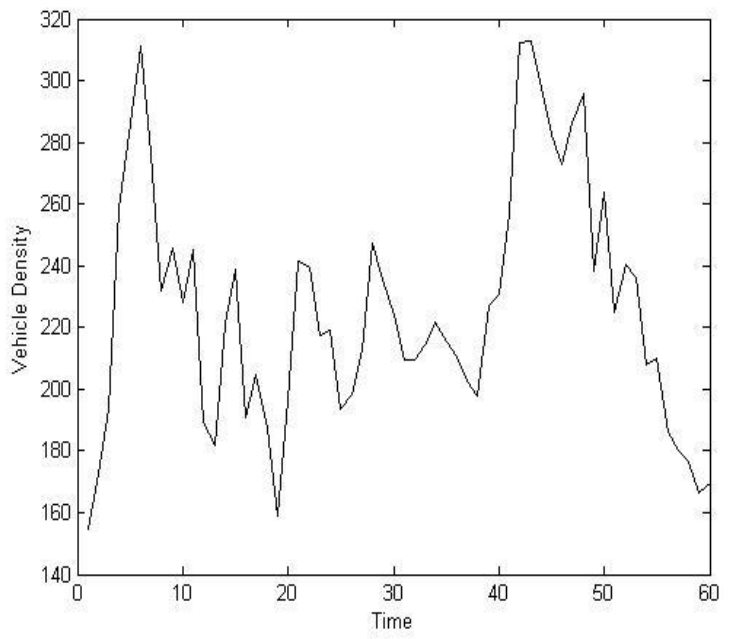

Fig. 1. Graphic of Vehicles Density in KS Tubun Street.

By the graphic, we can see that at the beginning of observation from 06.00 to 21.00 , the vehicle density is increase from 150 ucp to 310 ucp. The vehicle density in KS Tubun street tend to be stable on the $10^{\text {th }}$ until $40^{\text {th }}$ observations, but increaseon the $45^{\text {th }}$ to $50^{\text {th }}$ observation, and then decrease on the $60^{\text {th }}$ observation. The graphic of vehicles density is used as an origin value of vehicle density, which is 226 ucp at $\mathrm{t}=0$.

Table 2. Conversion Data

\begin{tabular}{|c|c|c|c|c|c|c|c|}
\hline Time & MC & LV & HV & Time & MC & LV & HV \\
\hline $06.00-06.15$ & 61.65 & 87 & 6 & $09.00-09.15$ & 81.90 & 94 & 6 \\
\hline $06.15-06.30$ & 80.40 & 91 & 1.5 & $09.15-09.30$ & 97.65 & 116 & 7.5 \\
\hline $06.30-06.45$ & 68.10 & 121 & 4.5 & $09.30-09.45$ & 92.85 & 131 & 15 \\
\hline $06.45-07.00$ & 102.15 & 149 & 7.5 & $09.45-10.00$ & 91.05 & 88 & 12 \\
\hline $07.00-07.15$ & 105.60 & 167 & 13.5 & $10.00-10.15$ & 92.70 & 94 & 18 \\
\hline $07.15-07.30$ & 115.80 & 188 & 7.5 & $10.15-10.30$ & 89.70 & 81 & 16.5 \\
\hline $07.30-07.45$ & 100.35 & 167 & 9 & $10.30-10.45$ & 73.95 & 76 & 9 \\
\hline $07.45-08.00$ & 102.15 & 119 & 10.5 & $10.45-11.00$ & 83.85 & 101 & 12 \\
\hline $08.00-08.15$ & 94.95 & 134 & 16.5 & $11.00-11.15$ & 89.10 & 133 & 19.5 \\
\hline $08.15-08.30$ & 92.85 & 122 & 13.5 & $11.15-11.30$ & 95.10 & 141 & 3 \\
\hline $08.30-08.45$ & 78.15 & 146 & 21 & $11.30-11.45$ & 88.65 & 123 & 6 \\
\hline $08.45-09.00$ & 76.80 & 102 & 10.5 & $11.45-12.00$ & 88.65 & 119 & 9 \\
\hline
\end{tabular}

2. The simulation of traffic flow in KS Tubun street based on model Greenberg modelcan be seen in Fig. 2.

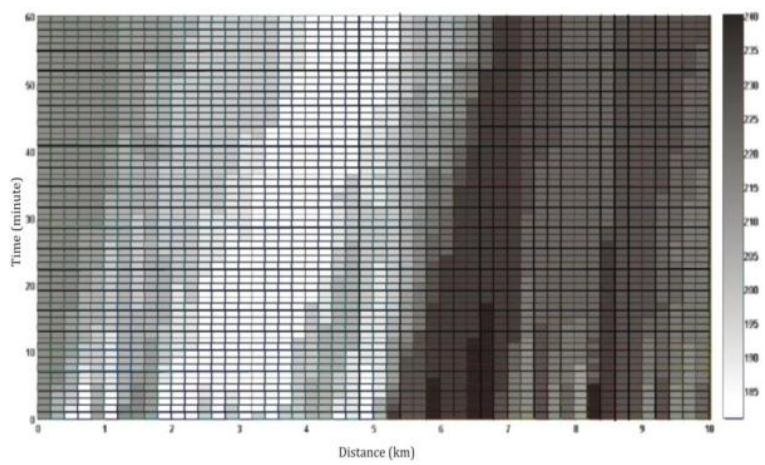

Fig. 2. Vehicle Density in KS Tubun street by Greenberg model

In Fig 2, the white color indicates the vehicle density is 180-205 ucp, the soft grey color indicates the vehicle density is $210-215$ ucp, the dark grey color indicates the vehicle density is 220-240 ucp. If the white color is fader than before, it means that the vehicle density is increase. Whereas, if the black color is more contrast, it means that the vehicle density will increase. The vehicle density is fluctuate between $180-210$ ucp on $0-5 \mathrm{~km}$, increased by $210-240$ ucp on $7-10$ $\mathrm{km}$. based on the color is fade, the graphic of vehicle density can be break down by the increasing of distance on $10 \mathrm{~km}$.

3. The simulation of vehicle velocity based on Greenberg model. The result can be seen in Fig. 3 .

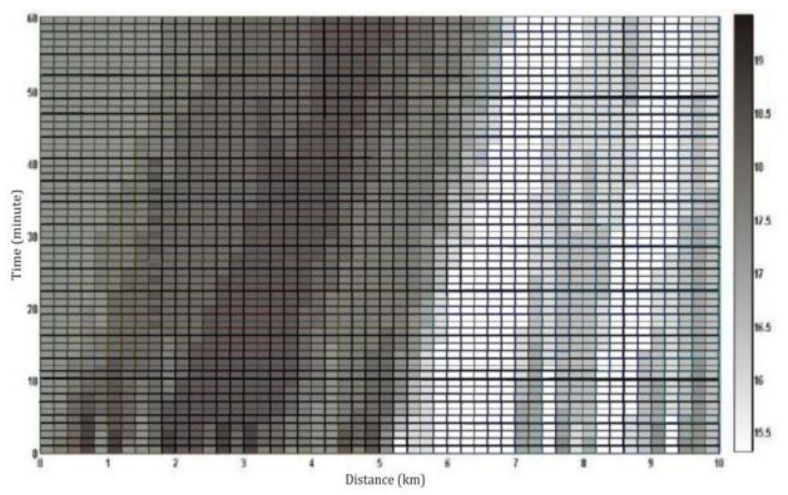

Fig. 3. Vehicle velocity in KS Tubun street based on Greenberg Model

In Fig. 3, the white color indicates the vehicle velocity is $15-17 \mathrm{~km} /$ hour, The grey color indicates the vehicle velocity is $17-17.5 \mathrm{~km} /$ hour, The dark grey color indicates the vehicle velocity is $17.5-18 \mathrm{~km} /$ hour, the black color indicates the vehicle velocity is $18-19.5 \mathrm{~km} /$ hour, if the white color is fader than before, it means that the velocity is increase, whereas if the black color is contrast, it means that the vehicle velocity will increase. The vehicle velocity is fluctuate between $17.5-19.5 \mathrm{~km} /$ hour with the distance is $0-5 \mathrm{~km}$. In $0-10 \mathrm{~km}$ on distance, the velocity tend to decrease. It can be seen from the changing of the color, from black color to be white color. The vehicle velocity can be increase or decrease from the first observation but tend to be constant follow the increase of distance. Based on the graphic, the vehicle velocity is increase on $10^{\text {th }}$ minute. 
The comparison of density and velocity of the vehicles before and after using Greenberg model can be seen at table 3 as follow :

Table 3. The Comparison Density and Velocity of Traffic Flow in KS. Tubun Street.

\begin{tabular}{|c|c|c|}
\hline & $\begin{array}{c}\text { Before using } \\
\text { Greenberg Model }\end{array}$ & $\begin{array}{c}\text { After using } \\
\text { Greenberg Model }\end{array}$ \\
\hline Density (ucp) & $150-310$ & $180-240$ \\
\hline Velocity (km/hour) & $10-20$ & $15-19.5$ \\
\hline
\end{tabular}

\section{Determining the waiting Time of Vehicles in Traffic Lights Queues}

According to Rouphail et al (2001) in deterministic concept, there are several components that must be considered to determine the waiting time in the queue of vehicles at the intersection traffic lights at a particular time duration [2], those are:

a. The existence of uniformity of patterns of the average vehicle's arrival for a period. The pattern of arrival of vehicles is not dependent to the time.

b. The arrival of the vehicle does not exceed the capacity specified by the maximum number of vehicles in the queue.

c. In the early phase of the green light, the entire vehicle in the queue just started moving left the queue.

d. The existence of uniformity of patterns of departure of a vehicle when it left the queue.

According to khabir at al (2010), the total waiting time in the queue of vehicles traffic in a single traffic light cycle during the red light and green light phase in equation below [3]:

$$
d=\frac{1}{2} r(1-\rho)^{-1}\left(\frac{2}{\lambda} E(Q(0))+R+\frac{1}{\mu}\left(1+I(1-\rho)^{-1}\right)\right.
$$

with $\mathrm{Q}(\mathrm{t})$ is the number of vehicles in the queue at time $\mathrm{t}$, $(0 \leq \mathrm{t} \leq \mathrm{T}), \lambda$ is the average vehicle that entered into the queue per second and $\mu$ is the average vehicles out leaving the queue per second, if the waiting time of vehicles that enter into queue, noted by $\mathrm{T}$ or time of traffic cycle and waiting time of vehicles which leave the queue is noted by the glowing green light duration (T-R).

The waiting time in the queue of vehicles in KS Tubun Street is obtained by using data of DLLAJ Bogor. With $\lambda=$ $992 \mathrm{ucp} /$ hour, long a cycle $\mathrm{T}=150$ minutes long, the green light turns on $(\mathrm{G})=60$ seconds, the number of vehicles leaving the queue $\mu=2873 \mathrm{ucp} /$ hour. After converted into seconds, then retrieved $\lambda=0.2755 \mathrm{ucp} /$ second, $\mu=0.7981$ ucp/second. The length of a glowing red light can be obtained by reducing the cycle time a traffic light with a long lit the green light, and obtained $\mathrm{R}=90$ seconds. The rest of the vehicles in the previous cycle $(\mathrm{Q}(0))$ are obtained from the data is $30 \mathrm{ucp}$. The waiting time in the queue of vehicles as follows:

$$
d=\frac{1}{2} r(1-\rho)^{-1}\left(\frac{2}{\lambda} E(Q(0))+R+\frac{1}{\mu}\left(1+I(1-\rho)^{-1}\right)\right.
$$

$$
\begin{aligned}
& =\frac{1}{2}\left(\frac{R}{T}\right)\left(1-\frac{\lambda}{\mu}\right)^{-1}\left(\frac{2}{\lambda} E(Q(0))+R+\frac{1}{\mu}\left(1+I\left(1-\frac{\lambda}{\mu}\right)^{-1}\right)\right. \\
& =\frac{1}{2}\left(\frac{90}{150}\right)\left(1-\frac{0.2755}{0.7981}\right)^{-1}\left(\frac{2 \times 30}{0.2755}+90+\frac{1}{0.7981}\left(1+0.1762\left(1-\frac{0.2755}{0.7981}\right)^{-1}\right)\right. \\
& =141.71 \approx 142 \text { second. }
\end{aligned}
$$

The waiting time in the queue red light vehicles can be found by dividing the total waiting time in the queue during the entire vehicle red light $(\mathrm{R})$ divided by the number of vehicles leaving the queue during the green light which can be written in the following equation:

$$
\begin{aligned}
d_{R} & =\frac{E[Q(0)] R+\frac{1}{2} \lambda R^{2}}{\lambda G}=\frac{30 \times 90+\frac{1}{2} 0.2755 \times 90^{2}}{0.7981 \times 60} \\
& =79.6846 \approx 80 \text { second. }
\end{aligned}
$$

Based on those results can be obtained an average waiting time of vehicles during the green lights is reducing the total waiting time vehicle during one cycle of the traffic light with the waiting time of the vehicle as long as the red light phase, thus the waiting time in the queue of vehicles traffic during this green light phase was 62 seconds. The waiting time in the queue of vehicles traffic lights can also be interpreted as the length of light up of traffic lights phase.

Table 4 will show the comparison table of the waiting time vehicles on KS. Tubun Street before and after using the Greenberg model.

Table 4. The Comparison of Waiting Time in KS. Tubun Street.

\begin{tabular}{|c|c|c|}
\hline $\begin{array}{c}\text { Traffic } \\
\text { Light }\end{array}$ & $\begin{array}{c}\text { The waiting Time of Vehicles } \\
\text { In KS Tubun Street before using } \\
\text { Greenberg Model }\end{array}$ & $\begin{array}{c}\text { The waiting Time of Vehicles } \\
\text { In KS Tubun Street After using } \\
\text { Greenberg Model }\end{array}$ \\
\hline Red & 90 second & 80 second \\
\hline Green & 60 second & 62 second \\
\hline Total & 150 second & 142 second \\
\hline
\end{tabular}

Based on Table 3, the waiting time of a vehicle at a traffic light in KS. Tubun Street by applied the queuing theory and Greenberg model, obtained the total time the traffic light can be minimized into 142 seconds, with a red light on for 80 minutes and the green light turns on during the time of 62 seconds, it also seen that the time the red light is lit on the traffic light cycle is reduced for 10 seconds, so the long queues of vehicles in the queue of the traffic light in KS. Tubun street can be reduced.

\section{CONCLUSION}

Traffic flow on the road KS Tubun street can be optimized by using Greenberg model as follows:

1. The density of vehicles in KS. Tubun Street before using Greenberg model is 150 to 310 unit of car passenger (ucp), whereas after using the Greenberg model, the vehicle density is 180 to 240 unit of car passenger.

2. between 15 to $19.5 \mathrm{~km}$ per hour. The density of vehicles happened in KS. Tubun street based on the Greenberg model can be break down slowly with increasing of time.

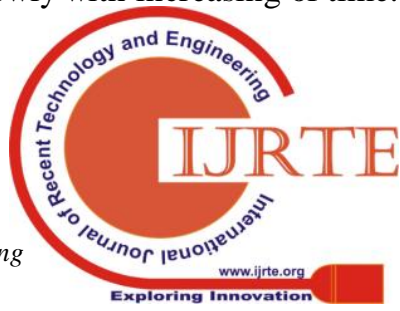


The waiting time of a vehicle on a traffic light during the red light and green light phase, obtained that the time of the red light on during 80 minutes and time is turned on the green light for 62 seconds with a total waiting time of the vehicle for one traffic light cycle is during 142 seconds. The waiting time in the queue of vehicles traffic lights based on Greenberg model can reduce the waiting time in the queue of the traffic light for 8 seconds.

\section{REFERENCES}

1. P. Kachroo and S. Sastry, Traffic Flow Theory Mathematical Framework, California: University of California Berkeley Press. 2012.

2. N. Rouphail, A. Tarco and J. Li, Traffic Flow at Signalized Intersection, Traffic Flow Theory Monograph, Indiana: Purdue University. 2001

3. A. Khabir Gani. "Numerical Simulation of A Mathematical Traffic Flow Model Based On A Nonlinear Velocity-Density Function”, Journal of Bangladesh Academy of Sciences, vol. 34. 2015.

4. O. R Jaimes , L. AQ. Tellez and G. F. Anaya Stability Analysis In Greenberg Traffic Model. International Journal of Modern physics. Vol 28. 2017.

5. G. Amandou, M. Lyudmila, and B Rene. Intermacrosopic Model for Traffic Networks. IEEE Transaction On Intelligent Tranfortation System. Vol 11. 2010.

6. B.S. Kerner. The Physics Of Traffic Empirical Free Way Patteren Features. Engineering Applications And Theory. Springer. 